\title{
Validação de Materiais Educativos para Orientação de Pacientes em Tratamento de Câncer de Mama com Hormonioterapia
}

\author{
doi: https://doi.org/10.32635/2176-9745.rbc.2020v66n4.1079 \\ Validation of Educational Materials to Guide Patients on Breast Cancer Treatment with Hormone Therapy \\ Validación de Materiales Educativos para Orientación de Pacientes en Tratamiento de Cáncer de Mama con Terapia Hormonal
}

Ana Carolina Anversa Sugisaka'; Vânia Mari Salvi Andrzejevski²; Inajara Rotta

\begin{abstract}
RESUMO
Introduçáo: A baixa adesão ao tratamento com antineoplásicos orais reflete a necessidade de medidas que facilitem o cumprimento da terapia, como a provisão de materiais educativos. Objetivo: Desenvolver e validar cartilhas orientando o uso dos medicamentos tamoxifeno, anastrozol e capecitabina no tratamento do câncer de mama. Método: Para a definiçáo do conteúdo das cartilhas, foram consultadas as bases de dados MEDLINE e UpToDate, e as diretrizes nacionais e internacionais, adotando-se linguagem acessível ao público. A validação foi feita por especialistas em oncologia, por meio do método Delphi, considerando-se os atributos de linguagem, conteúdo e aparência. Um consenso satisfatório foi atingido quando o valor de faixa interquartil (FIQ) foi $\leq 1$, obtido por meio de uma escala Likert variando de 1 a 5. Resultados: O grupo de especialistas foi composto por 29 profissionais. Na primeira rodada, foi obtida uma FIQ de 1,43; 1,14 e 1,43; e, na segunda rodada, 0,71; 1,04 e 0,79 para linguagem, conteúdo e aparência, respectivamente. Conclusáo: Espera-se que essas ferramentas promovam melhor percepção e adesão do paciente ao tratamento, contribuindo para a autogestão da farmacoterapia. Palavras-chave: Neoplasias da Mama/tratamento farmacológico; Estudos de Validação; Educação em Saúde; Materiais Educativos; Assistência Farmacêutica.
\end{abstract}

\section{ABSTRACT}

Introduction: The low adherence to treatment with oral antineoplastic agents reflects the need for measures to facilitate compliance with therapy, such as providing educational materials. Objective: Develop and validate leaflets guiding the use of tamoxifen, anastrozole and capecitabine for breast cancer treatment. Method: To define the leaflet's content, the MEDLINE and UpToDate databases and national and international guidelines were consulted, adopting language accessible to the public. The validation was performed by oncology specialists through the Delphi method, considering the attributes of language, content and appearance. A satisfactory consensus was reached when the interquartile range (IR) was $\leq 1$, obtained through a Likert scale ranging from 1 to 5 . Results: The group of specialists was formed by 29 professionals. In the first round an IR of 1.43 was obtained; 1.14 and 1.43 , and, in the second round, $0.71 ; 1.04$ and 0.79 for language, content and appearance, respectively. Conclusion: It is expected that these tools promote better patient perception and adherence to treatment, contributing to self-management of pharmacotherapy.

Key words: Breast Neoplasms/drug therapy; Validation Study; Health Education; Educational and Promotional Materials; Pharmaceutical Services.

\section{RESUMEN}

Introducción: La baja adherencia al tratamiento con agentes antineoplásicos orales refleja la necesidad de medidas para facilitar el cumplimiento de la terapia, como la provisión de materiales educativos. Objetivo: Desarrollar y validar folletos que guíen el uso de tamoxifeno, anastrozol y capecitabina en el tratamiento de cáncer de mama. Método: Para definir el contenido de los folletos, se consultaron las bases de datos MEDLINE y UpToDate, además de guías nacionales y internacionales, adoptando lenguaje accesible al público. La validación fue realizada por especialistas en oncología, a través del Método Delphi, considerando los atributos de lenguaje, contenido y apariencia. Se alcanzó un consenso satisfactorio cuando el rango intercuartil (RI) fue $\leq 1$, obtenido utilizando escala Likert que varia de 1 a 5 . Resultados: El grupo de expertos fue compuesto por 29 profesionales. En la primera ronda, se obtuvo um RI de 1.43; 1.14 y 1.43 , y, en la segunda, $0.71 ; 1.04$ y 0.79 para lenguaje, contenido y apariencia, respectivamente. Conclusión: Se espera que estas herramientas promuevan mejor percepción del paciente y adherencia al tratamiento, contribuyendo para el autocontrol de la farmacoterapia.

Palabras clave: Neoplasias de la Mama/tratamiento farmacológico; Estudios de Validación; Educación en Salud; Materiales Educativos y de Divulgación; Servicios Farmacéuticos.

${ }^{1}$ Centro Integrado de Oncologia de Curitiba (Cionc). Curitiba (PR), Brasil. Orcid iD: https://orcid.org/0000-0001-8294-0984

${ }^{2}$ Complexo Hospital de Clínicas da Universidade Federal do Paraná (CHC/UFPR). Curitiba (PR), Brasil. Orcid iD: https://orcid.org/0000-0003-3877-1487

${ }^{3}$ CHC/UFPR. Curitiba (PR), Brasil. Orcid iD: https://orcid.org/0000-0002-9099-7216

Endereço para correspondência: Inajara Rotta. Rua General Carneiro, 181 - Alto da Glória. Curitiba (PR), Brasil. CEP 80060-900. E-mail: inarotta@gmail.com 


\section{INTRODUÇÃO}

O câncer possui relevância epidemiológica, social e econômica, sendo um dos principais problemas de saúde pública no mundo. Nesse contexto, o câncer de mama representa o tipo mais prevalente entre as mulheres em todo o mundo, tendo representado $24,2 \%$ dos casos no ano de $2018^{1}$. No Brasil, de acordo com o Instituto Nacional do Câncer José Alencar Gomes da Silva (INCA), a estimativa de incidência é de aproximadamente 66.280 casos novos de câncer de mama para cada ano do triênio 2020-2022 $2^{2}$.

$\mathrm{O}$ tratamento atual combina abordagem local e sistêmica. A abordagem sistêmica inclui medicamentos administrados pela via endovenosa ou oral. Entre os orais, há aqueles enquadrados como terapia-alvo, que é o caso da hormonioterapia. A hormonioterapia é indicada para pacientes com receptores de hormônio positivos e consiste em um tratamento contínuo com tamoxifeno, um antiestrogênico, ou anastrozol, um inibidor de aromatase, sendo que a duraçáo do tratamento pode variar entre cinco e dez anos e está relacionada com a melhora da sobrevida livre da doença e da taxa de mortalidade. A hormonioterapia possui bons resultados e acessibilidade pelo Sistema Único de Saúde (SUS) ${ }^{3-5}$. Outro medicamento utilizado pela via oral é a capecitabina, um quimioterápico indicado para câncer de mama metastático ou como terapia adjuvante em pacientes com doença residual; sua utilização é fracionada, com regime terapêutico de 14 dias, seguido de sete dias de descanso e pode variar de três a oito ciclos. Esse regime posológico torna a farmacoterapia mais complexa, podendo comprometer a adesão pelo paciente ${ }^{6}$.

Nas últimas décadas, aumentou significativamente o uso de medicamentos orais no tratamento do câncer, trazendo inúmeras vantagens, como menor risco de infecção e de desconforto ao paciente, pelo fato de ser uma via não invasiva, e redução no número de visitas ao centro de tratamento, aumentando sua independência e evitando que sua rotina habitual seja afetada ${ }^{7-9}$. Uma revisão da literatura, que incluiu 13 estudos sobre a preferência dos pacientes em relação à via de administração dos quimioterápicos, encontrou uma maior preferência pela via oral em 11 estudos e afirmou que as principais razóes para isso são maior comodidade, qualidade de vida e autonomia ${ }^{10}$. Ao mesmo tempo, como todo medicamento, a terapia oral do câncer também apresenta desvantagens, como risco de superdosagem e necessidade de um maior autocuidado ${ }^{8,9}$.

Para que o paciente possa fazer o tratamento de forma segura e efetiva, é necessário que exista uma boa adesão à farmacoterapia. A possibilidade de não adesão ao tratamento ou, até mesmo, sua interrupção, é uma preocupação crescente entre os profissionais de saúde.
Esses fatos podem estar associados à crença, por parte do paciente, de que o tratamento não é mais necessário ou em decorrência do aparecimento de reaçôes adversas. Ainda, de forma mais frequente, a não adesão pode ser involuntária, resultado de esquecimento, problemas físicos ou cognitivos, ou falta de compreensão sobre o tratamento ${ }^{11-13}$.

Desse modo, a adesão em conjunto com o acesso e a qualidade da assistência ao paciente são fatores importantes para atingir bons resultados no enfrentamento da doença. Porém, estudos demonstram que as taxas de adesão aos antineoplásicos orais podem ser inferiores a $50 \%$ e que tendem a ser ainda menores ao longo do tratamento $^{14}$. Estudos que avaliaram a adesão específica à hormonioterapia adjuvante encontraram taxas de 80 $87 \%$ para o tamoxifeno e $69-88 \%$ para o anastrozol ${ }^{15,16}$. A não adesão à farmacoterapia reflete na efetividade do tratamento, progressão da doença, aumento dos custos e da demanda pelos serviços de saúde ${ }^{14,17}$.

Materiais educativos são importantes ferramentas na educação em saúde, complementando e reforçando as orientaçôes verbalizadas, visando ao aumento do conhecimento do paciente sobre a sua farmacoterapia e, como consequência, a uma maior adesão e autocuidado. É fundamental que, antes de serem utilizados, esses materiais sejam validados por meio de julgamento de especialistas com experiência na área, bem como da população a que se destinam. A validação pelos especialistas é uma etapa necessária, a fim de assegurar a cientificidade do conteúdo apresentado $^{18,19}$.

Dessa forma, este estudo apresenta o processo de construção e validação de materiais educativos, orientando o uso dos medicamentos tamoxifeno, anastrozol e capecitabina no tratamento do câncer de mama.

\section{MÉTODO}

Trata-se de uma pesquisa qualitativa metodológica, conduzida em um hospital público universitário. O estudo foi realizado em duas etapas: a) construção de cartilhas educativas contendo informaçóes aos pacientes em início de tratamento de câncer de mama com os antineoplásicos orais tamoxifeno, anastrozol e capecitabina; e b) validação destas por meio de um consenso entre juízes especialistas na área.

As cartilhas foram desenvolvidas de acordo com recomendaçôes para construção e avaliação de materiais educativos, considerando os aspectos de conteúdo, linguagem, ilustraçôes, layout e design $n^{20,21}$. Para a definição do conteúdo, foram consultadas as bases de dados MEDLINE/PubMed e UpToDate, assim como Diretrizes de Sociedades Brasileiras, Americanas e Europeias. A 
linguagem utilizada foi adaptada de forma a ser de fácil compreensão pelo paciente. $\mathrm{O}$ trabalho de design e diagramação das imagens foi feito por profissional da Unidade de Comunicação da Instituição.

Em uma segunda etapa, esses materiais foram validados por especialistas na área de oncologia, por meio do método Delphi. Essa técnica possui como objetivo obter o máximo de consenso de um grupo de especialistas, sobre um determinado tema, quando a unanimidade de opinião não existe, em virtude da falta de evidências científicas ${ }^{22}$.

Para a seleção dos juízes, foram considerados os seguintes critérios: serem farmacêuticos, médicos ou enfermeiros com atuaçáo na área da oncologia por no mínimo um ano. Inicialmente, foram convidados residentes, profissionais e chefes de serviços da instituição. A seguir, foi utilizado o método de bola de neve para seleção de novos especialistas, em que os juízes previamente selecionados indicaram outros participantes ${ }^{23}$. Para o contato com os especialistas, foi utilizado o correio eletrônico. Os questionários foram elaborados por meio do Google Formulários, permitindo que os participantes respondessem de forma a manter o anonimato e a distância entre pesquisadores e especialistas.

O processo de consulta da opinião dos especialistas ocorreu por meio de rodadas de perguntas até ser atingido um determinado nível de concordância entre eles. No primeiro momento, foi encaminhada uma carta de apresentaçáo do projeto e seus objetivos, convidando-os a participarem do estudo, juntamente com um questionário e os materiais educativos, em sua primeira versão, para serem analisados. $\mathrm{O}$ questionário foi subdividido em: a) Identificaçáo e informações gerais sobre os especialistas, como: nome, idade, número do registro profissional, área de atuaçáo, telefone e qualificaçōes; b) Questionário semiestruturado contendo nove questóes, sendo três fechadas e seis abertas. As questôes fechadas avaliaram os aspectos de linguagem, conteúdo e aparência dos materiais e continham notas variando de 1 a 5 na escala Likert, sendo 1 a pior nota, indicativo de falhas no desenvolvimento das cartilhas, e 5 a maior nota. As questóes abertas permitiram que os participantes sugerissem modificaçôes julgadas necessárias para melhorar os aspectos abordados nas questôes fechadas.

Com a devolutiva dos participantes, as respostas foram planilhadas no software Excel 2016 e as sugestóes, registradas nos campos abertos, avaliadas, tendo como base a contribuição que poderiam trazer para a pesquisa, sendo incluídas nos materiais em análise. Após a realização das modificaçôes, os especialistas receberam um feedback, tendo sido assegurado o sigilo da autoria das opinióes, juntamente com a segunda versão do material educativo, dando início, dessa forma, à segunda rodada de avaliaçáo dos materiais.
Para a segunda rodada, foi encaminhado o questionário modificado contendo seis questóes, sendo quatro fechadas e duas abertas. As quatro questóes fechadas continham notas variando de 1 a 5 , repetindo-se a avaliação dos três aspectos da primeira rodada (conteúdo, linguagem e aparência). Com base nos questionários da segunda rodada, as respostas foram avaliadas e as modificaçóes realizadas, seguindo os mesmos critérios da primeira.

As três questôes fechadas avaliando linguagem, conteúdo e aparência, comuns aos dois questionários, foram comparadas com o objetivo de verificar se existiam diferenças significativas entre as notas dos especialistas nas duas rodadas. Para tanto, foi realizado o teste de Wilcoxon pareado no software SPSS v.19, sendo adotado um nível de 5\% de significância. Além disso, para definir um consenso entre os especialistas, dentro da mesma rodada, foi realizado um cálculo de faixa interquartil (FIQ). Um consenso satisfatório foi atingido quando o valor de FIQ encontrado foi $\leq 1$.

As questôes abertas foram analisadas e as respostas foram classificadas como "Sim" ou "Não". As respostas "Sim" foram adotadas quando houve sugestáo do especialista dentro da rodada e "Náo" na ausência de sugestóes de modificação do material analisado. Posteriormente, foi avaliado se houve diferença significativa na proporção de respostas "Sim" ou "Náo", comparando a primeira e a segunda rodadas. Para a análise estatística, foram utilizados os softwares Excel 2016 e Action Stat v.3.2, sendo realizado o teste para duas proporçôes, adotando um nível de 5\% de significância.

O estudo seguiu princípios éticos que comandam as pesquisas com seres humanos, segundo a resolução n.o 466/2012, sendo aprovado pelo Comitê de Ética em Pesquisa da Instituição (CAAE: 61351016.9.0000.0096). Como condiçáo para participação, todos os especialistas assinaram o Termo de Consentimento Livre e Esclarecido.

\section{RESULTADOS}

\section{DESENVOLVIMENTO DOS MATERIAIS EDUCATIVOS}

O conteúdo consistiu em informaçóes acerca da apresentação do medicamento, administração, dose, armazenamento, reaçôes adversas mais comuns e condutas em caso de esquecimento de dose. O contato telefônico do Ambulatório Farmacêutico de Oncologia também foi informado, a fim de que o paciente tenha como contactar o farmacêutico, caso necessário. Na cartilha da capecitabina, foram acrescentadas informaçóes sobre a síndrome mão-pé, uma reação relativamente comum e que requer cuidados específicos. Na Figura 1, as cartilhas são apresentadas. 

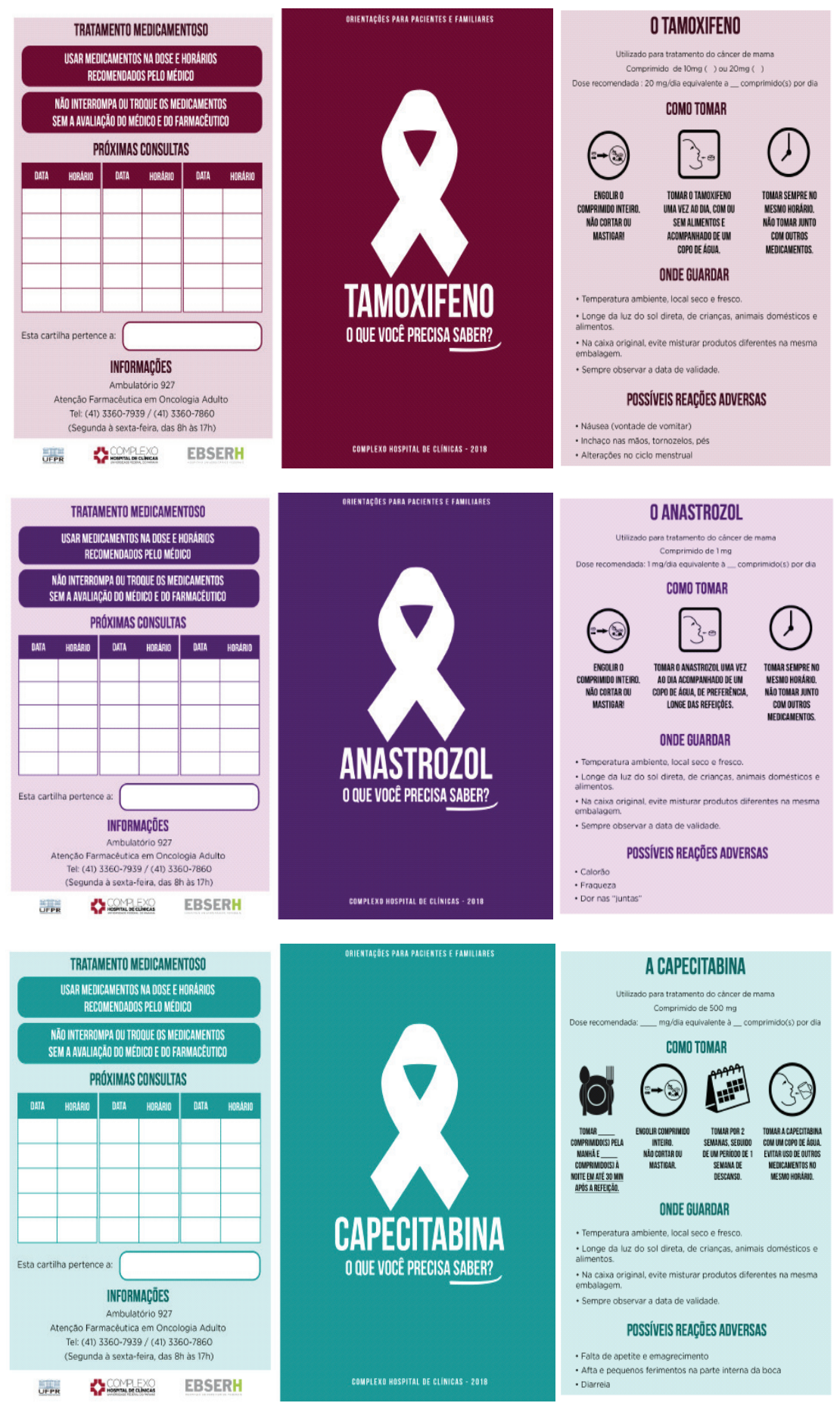
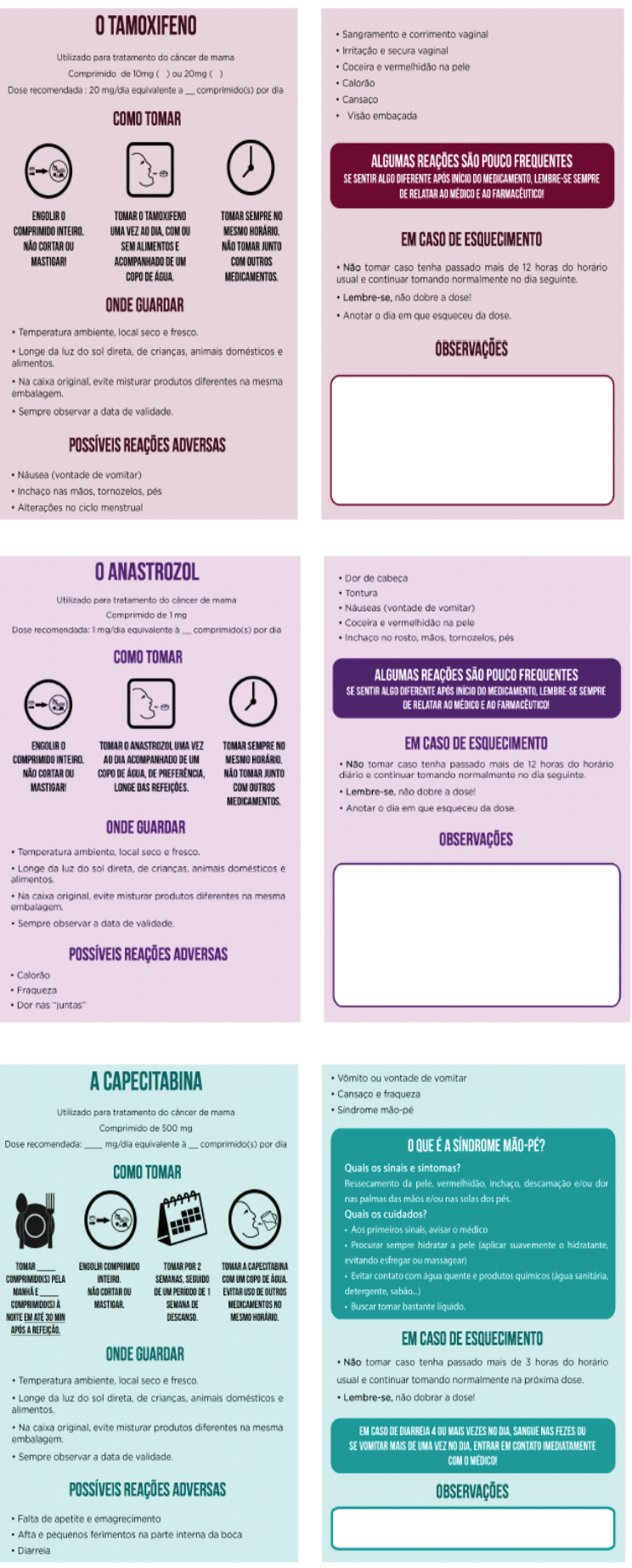

Figura 1. Cartilhas para orientação sobre o uso dos antineoplásicos tamoxifeno, anastrozol e capecitabina

\section{CARACTERIZAÇÃO DOS ESPECIALISTAS}

O grupo de especialistas foi composto por 30 profissionais de saúde: 16 farmacêuticos, sete enfermeiros e sete médicos. A idade dos profissionais variou entre 23 e 58 anos. Quinze profissionais possuíam de um a cinco anos de conclusão da graduação, sete, de seis a dez anos, cinco, de 11 a 15 anos e três, acima de 20 anos. Dos 30 participantes da pesquisa, 26 pertenciam a Centros de Assistência de Alta Complexidade em Oncologia (Cacon).

\section{RODADAS DE PERGUNTAS}

O nível de concordância desejado foi atingido em apenas duas rodadas. $\mathrm{Na}$ primeira rodada, os 30 especialistas responderam ao questionário. Sugestóes de alteraçóes de termos ou expressóes como "letargia" e "anorexia", apresentados na primeira versão dos materiais, foram as mais prevalentes. Além disso, foram realizadas sugestôes para acrescentar orientaçôes sobre a síndrome mão-pé, no material da capecitabina. Para melhorar a 
aparência, foram sugeridas alteraçóes nas figuras, cor e no layout.

Já na segunda rodada, 29 dos 30 especialistas responderam ao questionário. Foram realizadas sugestôes para melhorar a clareza na orientação em como utilizar o medicamento e na melhor conduta em caso de esquecimento. Além disso, alteraçôes nas cores também foram sugeridas para associar o câncer de mama ao Outubro Rosa. Alguns especialistas demonstraram que as modificaçôes realizadas, após a primeira rodada, foram satisfatórias.

\section{COMPARAÇÃO DOS RESULTADOS ENTRE AS RODADAS}

Comparando-se as questôes fechadas entre a primeira e a segunda rodadas, houve diferença estatisticamente significativa entre as medianas das notas dadas nos critérios de linguagem $(\mathrm{p}=0,004)$, conteúdo $(\mathrm{p}=0,039)$ e aparência $(\mathrm{p}=0,003)$.

No critério de linguagem, os especialistas avaliaram o grau de clareza e compreensão das informações com a linguagem adotada. Dos 29 avaliadores, dez atribuíram nota máxima na primeira rodada e 19 na segunda rodada, sendo observado um aumento de $32 \%$ na taxa de especialistas que conferiram nota 5 para o atributo. $\mathrm{Na}$ segunda rodada, as notas atribuídas variaram entre 4 e 5 (Figura 2).

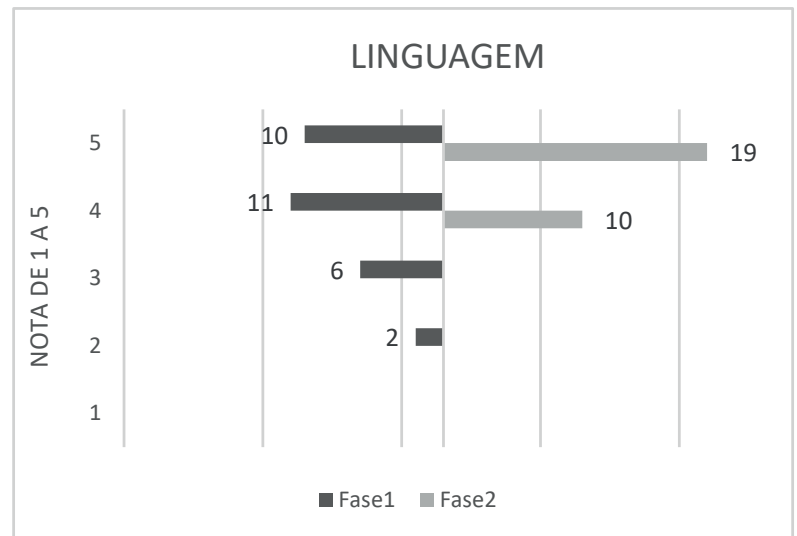

Figura 2. Notas dos especialistas atribuídas ao critério linguagem

O critério de conteúdo avaliava se as informaçóes presentes nos materiais eram suficientes para auxiliar o paciente caso ele tivesse alguma dúvida relacionada ao tratamento. Dos 29 avaliadores, nove atribuíram nota máxima na primeira rodada e 13 na segunda rodada, sendo observado um aumento de $11 \%$ na taxa de especialistas que conferiram nota 5 para o atributo. Na segunda rodada, as notas atribuídas variaram entre 3 e 5 (Figura 3).

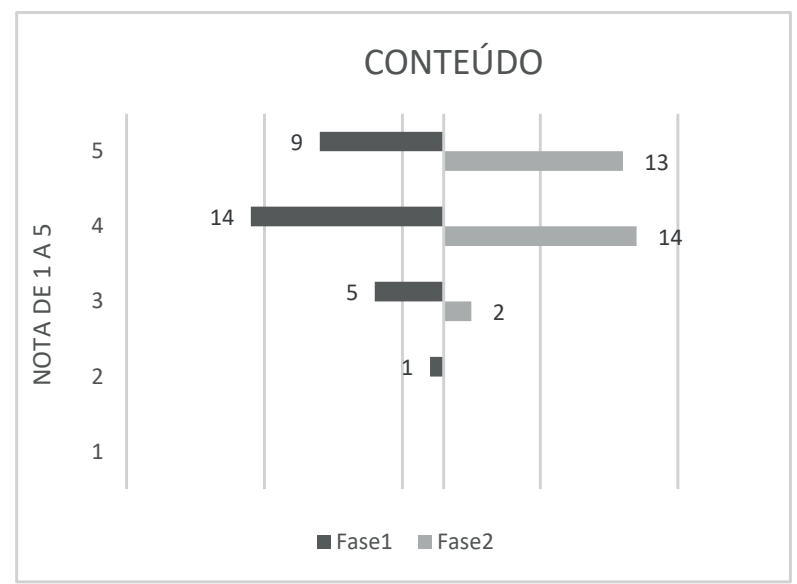

Figura 3. Notas dos especialistas atribuídas ao critério conteúdo

No critério de aparência, os especialistas avaliaram se os materiais eram visualmente atraentes. Dos 29 avaliadores, nove atribuíram nota máxima na primeira rodada e 18 na segunda rodada, sendo observado um aumento de $32 \%$ na taxa de especialistas que conferiram nota 5 para o atributo. $\mathrm{Na}$ segunda rodada, as notas atribuídas variaram entre 4 e 5 (Figura 4).

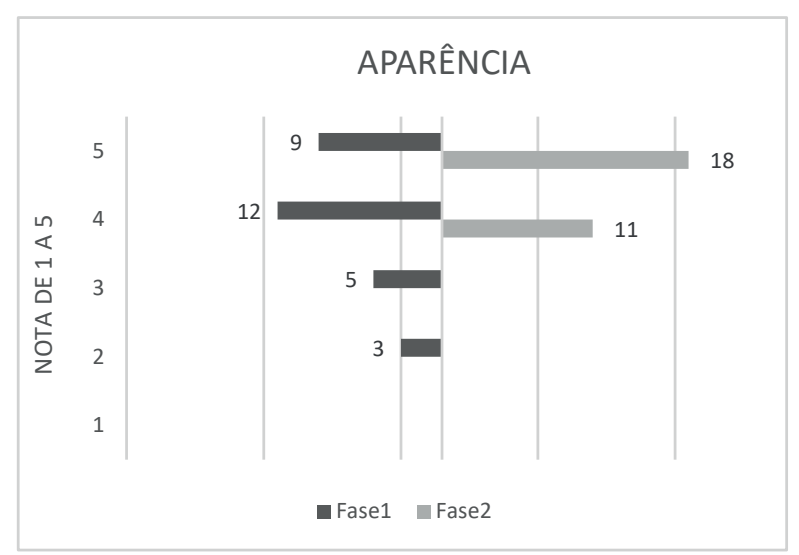

Figura 4. Notas dos especialistas atribuídas ao critério aparência

\section{CONSENSO DOS ESPECIALISTAS DENTRO DA RODADA}

A Tabela 1 representa o grau de consenso entre os especialistas calculado pela FIQ. Na primeira rodada, o resultado da FIQ foi maior do que 1 nos três aspectos avaliados. Já na segunda rodada, os resultados foram menores ou igual a 1 , sendo o consenso considerado satisfatório.

Quando comparadas as rodadas, houve diferença estatisticamente significativa na proporção de respostas "Sim" nas questóes abertas, o que indica a sugestáo de algum tipo de mudança nos materiais educativos, tendo 
Tabela 1. Valores de FIQ obtidos em cada rodada para os atributos linguagem, conteúdo e aparência

\begin{tabular}{c|c|c}
\hline & $\begin{array}{c}\text { FIQ } \\
\text { Rodada 1 }\end{array}$ & $\begin{array}{c}\text { FIQ } \\
\text { Rodada 2 }\end{array}$ \\
\hline Linguagem & 1,43 & 0,71 \\
Conteúdo & 1,14 & 1,04 \\
Aparência & 1,43 & 0,79 \\
\hline
\end{tabular}

25 especialistas sugerido alguma alteração na primeira fase e apenas dez na segunda $(\mathrm{p}<0,0001)$.

\section{DISCUSSÃO}

No ano de 2013, a American Society of Clinical Oncology (ASCO), em parceria com a Oncology Nursing Society (ONS), publicou um guia sobre a utilização segura dos antineoplásicos orais. Esse guia reforça que a adesão está correlacionada com o entendimento do paciente sobre seu regime terapêutico ${ }^{24}$. Desse modo, quanto maior o acesso a medicamentos orais, maior a necessidade de medidas que facilitem o cumprimento da terapia e, entre essas medidas, estão as orientaçóes em forma de materiais educativos impressos. O emprego de tecnologias educativas validadas reforça o processo de ensino-aprendizagem e estreita a comunicação entre o profissional de saúde e o paciente. Ainda, contribui para o empoderamento do paciente, promovendo o autocuidado e reduzindo os erros de medicaçáo, afetando positivamente os resultados terapêuticos ${ }^{18,19,25,26}$.

Nos últimos anos, houve uma transição do papel social do farmacêutico, deixando de ser responsável por atividades focadas apenas no medicamento, como manipulação e distribuição, passando a exercer funçóes voltadas ao cuidado ao paciente, participando de uma abordagem multidisciplinar junto com enfermeiros, médicos e outros profissionais da saúde ${ }^{27}$. A Lei n. ${ }^{\circ}$ $13.021 / 2014^{28}$, ao definir a Farmácia como uma unidade de prestação de serviço destinada a prover orientação sanitária individual e coletiva, reitera a importância da prática da educação em saúde pelos farmacêuticos. De acordo com o Conselho Federal de Farmácia (CFF), essa abordagem compreende diferentes estratégias educativas e tem como objetivo central promover a autonomia do paciente e o seu comprometimento com o tratamento ${ }^{29}$.

As atividades de provisão de informação, orientaçáo e educação do paciente, familiares e cuidadores sobre o uso racional de medicamentos, além da elaboração de materiais educativos, fazem parte das atribuiçôes clínicas do farmacêutico, segundo a Resoluçáo CFF n.o 585 de 29 de agosto de $2013^{30}$. Ainda de acordo com essa resoluçáo, o farmacêutico deve estabelecer um processo adequado de comunicação, que seja condizente com o letramento em saúde do receptor ${ }^{30}$. Dessa forma, neste estudo, foram propostos materiais educativos contendo informaçóes que os profissionais participantes consideraram de fácil compreensão pelo paciente, incluindo os horários e a forma correta de administração, o armazenamento, a melhor conduta em caso de esquecimento de dose e possíveis reaçóes adversas.

A técnica Delphi trouxe vantagens no processo de validação das cartilhas, pois permitiu a participação de 29 profissionais de diferentes áreas de conhecimento dentro de uma mesma especialidade, a Oncologia. Outrossim, o anonimato durante as rodadas possibilitou maior liberdade de expressáo aos participantes do estudo. Como a maior parte dos profissionais selecionados pertencia a Cacon, a validação dos materiais educativos tornou-se ainda mais efetiva. A forma de comunicação com os especialistas por meio de correio eletrônico tornou o estudo mais fácil de ser realizado e acarretou redução dos custos.

No processo de análise do conteúdo, linguagem e aparência das cartilhas, foram incluídas as contribuiçōes de todos os juízes, os quais sugeriram modificaçôes relevantes e consistentes. $\mathrm{Na}$ segunda rodada de perguntas, foram obtidas FIQ inferiores a 1 para os critérios de linguagem e aparência, e igual a 1 para o critério de conteúdo, não sendo necessária uma nova rodada. Ao final, os especialistas consideraram que as cartilhas apresentavam informaçôes completas e pertinentes, com linguagem clara, e eram visualmente atrativas, motivando a leitura.

Cabe destacar a importância da ação educativa presencial associada ao material escrito, sendo fundamental que tais formatos de intervençáo sejam complementares. Em estudos em que os materiais educativos foram apenas entregues ao paciente, sem nenhum tipo de orientação verbal, não houve melhora estatisticamente significativa dos resultados de adesão e persistência avaliados durante o período de 12 meses de seguimento de mulheres sob tratamento com hormonioterapia. Mesmo com os materiais sendo providos de forma regular, a ausência de comunicação verbal com o profissional de saúde não levou a resultados mais efetivos ${ }^{31,32}$. Já no estudo de Ziller et $\mathrm{al}^{25}$, as pacientes em uso de inibidores de aromatase que receberam orientaçóes por escrito e por telefone apresentaram melhores resultados de adesão e persistência durante o tempo de acompanhamento ( $\mathrm{p}=0,039)$.

Em atenção a isso, os pacientes acompanhados no Ambulatório Farmacêutico de Oncologia, em início de tratamento com tamoxifeno, anastrozol ou capecitabina, recebem esses materiais educativos de forma associada a uma explanação oral no momento da consulta farmacêutica e dispensaçáo do medicamento. No caso de 
pacientes que possuem cuidador, a provisão do serviço é destinada a ambos.

A não validação dos materiais educativos pelos pacientes beneficiários da intervenção farmacêutica, especialmente em relaçáo à sua compreensibilidade, aparência e impacto na promoção do seu conhecimento, consiste em uma limitaçáo do estudo, dada a importância de avaliar a eficiência das cartilhas considerando o julgamento do público-alvo. Entretanto, essa fragilidade será ratificada em estudo futuro, uma vez que já foi implementado um estudo-piloto, considerando a legitimação das cartilhas pelos pacientes acompanhados no Ambulatório Farmacêutico de Oncologia.

\section{CONCLUSÃO}

Os materiais educativos desenvolvidos, após as alteraçóes propostas pelos especialistas peritos em oncologia, mostraram-se atrativos, com linguagem clara e acessível, e com completude de informaçóes apropriadas, apresentando, portanto, potencial para a promoção do conhecimento e adesão do paciente ao tratamento, quando utilizados de forma associada à comunicação oral durante os atendimentos farmacêuticos.

Em etapa posterior, as cartilhas serão validadas pelos pacientes beneficiários da intervenção educativa, a fim de avaliar a sua aplicabilidade na prática.

\section{CONTRIBUIÇÕES}

Ana Carolina Anversa Sugisaka participou da concepção do estudo, coleta e análise dos dados, e na redação do artigo. Vânia Mari Salvi Andrzejevski participou da concepção e revisão do artigo. Inajara Rotta supervisionou todas as etapas do estudo e participou da análise dos dados e revisão crítica do artigo. Todas as autoras aprovaram a versão final a ser publicada.

\section{AGRADECIMENTOS}

A todos os especialistas que participaram do processo de validaçáo e ao Programa de Residência Multiprofissional em Atenção Hospitalar do Complexo Hospital de Clínicas da Universidade Federal do Paraná.

\section{DECLARAÇÃO DE CONFLITO DE INTERESSES}

Nada a declarar.

\section{FONTES DE FINANCIAMENTO}

Não há.

\section{REFERÊNCIAS}

1. Ferlay J, Ervik M, Lam F, et al. Global cancer observatory: cancer today [Internet]. Lyon (FR): International Agency for Research on Cancer; 2018 [cited 2020 May 11]. Available from: https://gco.iarc.fr/today

2. Instituto Nacional de Câncer José Alencar Gomes da Silva [Internet]. Rio de Janeiro: INCA; [data desconhecida]. Causas e prevençáo: estatísticas de câncer; [modificado 2020 maio 8; acesso 2020 maio 11]. Disponível em: https://www.inca.gov.br/numeros-de-cancer

3. Rugo HS, Rumble RB, Macrae E, et al. Endocrine therapy for hormone receptor-positive metastatic breast cancer: American Society of Clinical Oncology guideline. J Oncol Prac. 2016;34(25):3069-3103. doi: https://doi. org/10.1200/JCO.2016.67.1487

4. Burstein HJ, Temin S, Anderson H, et al. Adjuvant endocrine therapy for women with hormone receptorpositive breast cancer: American Society of Clinical Oncology clinical practice guideline focused update. J Clin Oncol. 2014;32(21):2255-69. doi: https://doi. org/10.1200/JCO.2013.54.2258

5. Ministério da Saúde (BR), Secretaria de Atenção à Saúde, Secretaria de Ciência, Tecnologia e Insumos Estratégicos. Portaria Conjunta no 19 , de 3 de julho de 2018. Aprova as Diretrizes Diagnósticas e Terapêuticas do Carcinoma de Mama [Internet]. Diário Oficial da União. 2018 jul 16; Seção 1:59 [acesso 2020 maio 12]. Disponível em: https://www.saude.gov.br/images/pdf/2018/julho/16/ Portaria-Conjunta-n-19--PCDT-Carcinoma-de-Mama. pdf

6. Simons S, Ringsdorf S, Braun M, et al. Enhancing adherence to capecitabine chemotherapy by means of multidisciplinary pharmaceutical care. Support Care Cancer. 2011;19(7):1009-18. doi: https://doi. org/10.1007/s00520-010-0927-5

7. Bergsbaken JJ, Eickhoff JC, Buss BA, et al. Assessment of adherence with oral anticancer agents in oncology clinical trials: a systematic review. J Oncol Pharm Pract. 2016;22(1):105-13. doi: https://doi. org/10.1177/1078155214567163

8. Barillet M, Prevost V, Joly F, et al. Oral antineoplastic agents: how do we care about adherence? Br J Clin Pharmacol. 2015;80(6):1289-1302. doi: https://doi. org/10.1111/bcp.12734

9. Rudnitzki T, McMahon D. Oral Agents for Cancer: safety challenges and recommendations. Clin J Oncol Nurs. 2015;19(3 Suppl):41-6. doi: https://doi.org/10.1188/15. S1.CJON.41-46

10. Eek D, Krohe M, Mazar I, et al. Patient-reported preferences for oral versus intravenous administration for the treatment of cancer: a review of the literature. Patient Prefer Adherence. 2016;10:1609-21. doi: https:// doi.org/10.2147/PPA.S106629 
11. Bourmaud A, Henin E, Tinquaut F, et al. Adherence to oral anticancer chemotherapy: what influences patients' over or non-adherence? Analysis of the OCTO study through quantitative-qualitative methods. BMC Res Notes. 2015;8:291. doi: https://doi.org/10.1186/ s13104-015-1231-8

12. Andrzejevski VMS. Fatores que podem influenciar na adesão aos antineoplásicos orais [dissertação]. Curitiba (PR): Setor de Ciências da Saúde, Universidade Federal do Paraná; 2016.

13. Correr CJ. Coleta e organização de dados do paciente. In: Correr CJ, Otuki MF, organizadores. A prática farmacêutica na farmácia comunitária. Porto Alegre: Artmed; 2013. p. 288-9.

14. Greer JA, Amoyal N, Nisotel L, et al. A systematic review of adherence to oral antineoplastic therapies. Oncologist. 2016;21(3):354-76. doi: https://doi.org/10.1634/ theoncologist.2015-0405

15. Ziller V, Kalder M, Albert US, et al. Adherence to adjuvant endocrine therapy in postmenopausal women with breast cancer. Ann Oncol. 2009;20(3):431-6. doi: https://doi.org/10.1093/annonc/mdn646

16. Arimidex, Tamoxifen, Alone or in Combination (ATAC) Trialists' Group; Forbes JF, Cuzick J, et al. Effect of anastrozole and tamoxifen as adjuvant treatment for early-stage breast cancer: 100-month analysis of the ATAC trial. Lancet Oncol. 2008;9(1):45-53. doi: https:// doi.org/10.1016/S1470-2045(07)70385-6

17. Verbrugghe M, Verhaeghe S, Lauwaert K, et al. Determinants and associated factors influencing medication adherence and persistence to oral anticancer drugs: a systematic review. Cancer Treat Rev. 2013;39(6):610-621. doi: https://doi.org/10.1016/j. ctrv.2012.12.014

18. McCue DA, Lohr LK, Pick AM. Improving adherence to oral cancer therapy in clinical practice. Pharmacotherapy. 2014;34(5):481-94. doi: https://doi.org/10.1002/ phar. 1399

19. Tadic D, Spasojevic IB, Tomasevic ZI, et al. Oral administration of antineoplastic agents: the challenges for healthcare professionals. J BUON. 2015;20(3):690-8.

20. Hoffmann T, Worrall L. Designing effective written health education materials: considerations for health professionals. Disabil Rehabil. 2004;26(19):116673. doi: https://doi.org/10.1080/09638280410001 724816

21. A Guide to creating and evaluating patient materials: guidelines for effective print communication [Internet]. Portland, ME: MaineHealth; 2010 Dec [cited 2020 May 7]. Available from: https://mainehealth.org/-/media/ community-education-program-cep/health-literacy/ mh-print-guidelines.pdf?la=en

22. Revorêdo LS, Maia RS, Torres GV, et al. O uso da técnica delphi em saúde: uma revisão integrativa de estudos brasileiros. Arq Ciên Saúde. 2015;22(2):16-21. doi: https://doi.org/10.17696/2318-3691.22.2.2015.136

23. Naderifar M, Goli H, Ghaljaie F. Snowball sampling: a purposeful method of sampling in qualitative research. Strides Dev Med Educ. 2017;14(3):e67670. doi: https:// doi.org/10.5812/sdme.67670

24. Neuss MN, Polovich M, McNiff K, et al. 2013 updated American Society of Clinical Oncology/Oncology Nursing Society chemotherapy administration safety standards including standards for the safe administration and management of oral chemotherapy. J Oncol Pract. 2013;9(2 Suppl):5s-13s. doi: https://doi.org/10.1200/ JOP.2013.000874

25. Ziller V, Kyvernitakis I, Knoll D, et al. Influence of a patient information program on adherence and persistence with an aromatase inhibitor in breast cancer treatment - the COMPAS study. BMC Cancer. 2013;13:407. doi: https://doi.org/10.1186/1471-240713-407

26. Zerillo J, Goldenberg B, Kotecha R, et al. Interventions to improve oral chemotherapy safety and quality: a systematic review. JAMA Oncol. 2018;4(1):105-117. doi: https://doi.org/10.1001/jamaoncol.2017.0625

27. Avery M, Williams F. The importance of pharmacist providing patient education in oncology. J Pharm Pract. 2015;28(1):26-30. doi: https://doi. org/10.1177/0897190014562382

28. Presidência da República (BR). Lei no 13.021, de 8 de agosto de 2014. Dispóe sobre o exercício e a fiscalização das atividades farmacêuticas [Internet]. Diário Oficial da União - Edição Extra. 2014 ago 11, Seção 1:1 [acesso 2020 maio 14]. Disponível em: https://www2.camara. leg.br/legin/fed/lei/2014/lei-13021-8-agosto-2014779151-normaatualizada-pl.html

29. Conselho Federal de Farmácia (BR). Serviços farmacêuticos diretamente destinados ao paciente, à família e à comunidade: contextualização e arcabouço conceitual. Brasília, DF: Conselho Federal de Farmácia; 2016.

30. Conselho Federal de Farmácia (BR). Resolução no 585, de 29 de agosto de 2013. Regulamenta as atribuiçóes clínicas do farmacêutico e dá outras providências. Diário Oficial da União, Poder Executivo, Brasília, DF. 2013. Diário Oficial da União. 2013 set 25; Seção 1:186 [acesso 2020 maio 13]. Disponível em: http://www.normaslegais.com. br/legislacao/Resolucao-cff-585-2013.htm

31. Hadji P, Blettner M, Harbeck N, et al. The Patient's Anastrozole Compliance to Therapy (PACT) Program: a randomized, in-practice study on the impact of a standardized information program on persistence and compliance to adjuvant endocrine therapy in postmenopausal women with early breast cancer. Ann Oncol. 2013;24(6):1505-12. doi: https://doi. org/10.1093/annonc/mds653 
32. Neven P, Markopoulos C, Tanner M, et al. The impact of educational materials on compliance and persistence rates with adjuvant aromatase inhibitor treatment: Firstyear results from the compliance of aromatase inhibitors assessment in daily practice through educational approach (CARIATIDE) study. Breast. 2014;23(4):39399. doi: https://doi.org/10.1016/j.breast.2014.02.009

Recebido em 2/6/2020 Aprovado em 2/7/2020 